\title{
Nonphysician Clinicians in the Follow-Up of Resected Patients with Colorectal Cancer
}

\author{
Robert R.J. Coebergh van den Braak ${ }^{a}$ Zarina S. Lalmahomed ${ }^{a} \quad$ Stefan Büttner ${ }^{a}$ \\ Bettina E. Hansen $^{b}$ Jan N.M. Ijzermans ${ }^{a}$ on behalf of the MATCH Study Group \\ ${ }^{a}$ Department of Surgery, and bepartment of Gastroenterology and Hepatology, Erasmus MC University Medical \\ Centre, Rotterdam, The Netherlands
}

\section{Keywords}

Colorectal cancer · Follow-up · Nonphysician clinicians ·

Survival analysis

\begin{abstract}
Background/Aims: The 5-year postoperative follow-up for patients undergoing curative treatment for colorectal cancer (CRC) is labour intensive. We assessed the added value of a dedicated nonphysician clinician (NPC) in the follow-up of patients after resection for CRC. Methods: Patients were divided into 2 groups as defined by the number of follow-up visits in the first year, including intensive $(\geq 3 \times)$ and minimal $(\leq 2 \times)$. Involvement of an NPC, diagnosis of disease recurrence and the course of the disease were determined. $\boldsymbol{R} \boldsymbol{e}$ sults: Of the 681 patients, $79.9 \%$ belonged to the "intensive" and $21.1 \%$ to the "minimal" group. Involvement of an NPC resulted in a higher adherence to follow-up (84.3 vs. $73.9 \%$, $p=0.001)$. Overall, patients in regular follow-up less often had multifocal recurrence (47.1 vs. $73.7 \%, p=0.04$ ), and a better survival after recurrence (SAR; hazard ratio [HR] 3.604, $p<0.001)$. The "intensive" group had a significantly better overall survival compared to the "minimal" group (HR 1.71, $p=0.013)$. Conclusion: Adherence to surveillance programs after resection for $C R C$ is better in hospitals with a dedicated NPC. Overall, patients' adherence to follow-up resulted in
\end{abstract}

\section{KARGER}

(C) 2017 S. Karger AG, Basel

E-Mail karger@karger.com

www.karger.com/ddi less multifocal disease recurrence at the time of diagnosis as compared to patients presenting with symptoms and a better 3-year SAR.

(c) 2017 S. Karger AG, Basel

\section{Introduction}

Colorectal cancer (CRC) is the third most common malignancy worldwide with over 1.2 million new cases surfacing annually and is therefore a major cause for higher rates of morbidity and mortality [1]. The main cause of death is metastatic disease [2]. Of all patients, 20-25\% will present with metastatic disease at the time of diagnosis and another 20-25\% will develop metastatic disease after curative treatment [2]. Therefore, patients undergoing curative treatment for CRC are followed for at least 5 years after surgery to detect recurrence. The 2 main components of the follow-up are blood carcinoembryogenic antigen measurement (CEA) and radiological imaging of the liver with CT imaging or ultrasound $[3,4]$. As the success of resection of liver metastases is increas-

R.R.J.C.B. and Z.S.L. contributed equally and should be acknowledged with first authorship. 
Table 1. Dutch surveillance program after curative resection of colorectal cancer

\begin{tabular}{llllll}
\hline & 1st year & 2nd year & 3th year & 4th year & 5th year \\
\hline 3 months & CEA & CEA & & & \\
\hline 6 months & CEA & CEA & CEA & CEA & CEA \\
& US/CT & US/CT & US/CT & & \\
\hline 9 months & CEA & CEA & & & CEA \\
\hline 12 months & CEA & CEA & CEA & CEA & US/CT \\
& US/CT & US/CT & US/CT & US/CT & \\
& Colonoscopy & & & & \\
\hline
\end{tabular}

ing with 5-year overall survival (OS) rates as high as $40-$ $60 \%$, early detection of liver lesions is warranted $[5,6]$.

Interestingly, although multiple follow-up regimens have been suggested in literature with differences in both frequency of follow-up and components used at various time points, the intensity and components of the followup are still a matter of debate $[7,8]$. A recent systematic review concluded that intensive follow-up strategies were associated neither with improved cancer-specific survival, nor with a higher detection rate for tumour recurrences after curative treatment of CRC [9]. The FACS-trial, the most recent randomized controlled trial comparing multiple follow-up strategies with the same intensity but different components, suggested that follow-up should include at least one of the 2 components, CEA or imaging [3].

The follow-up visits of patients with CRC is labour intensive because of both the administrative and logistic work that is required for a well-defined format. Therefore, nonphysician clinicians (NPCs) are increasingly employed to organize the follow-up of these patients. However, until now, no literature has been published assessing the added value of an NPC in the follow-up.

This study is the first of its kind to assess the added value of a dedicated PA in the follow-up of patients with CRC.

\section{Method}

All aspects of the Strengthening the Reporting of Observational studies in Epidemiology (STROBE) guidelines were followed, and the paper was written accordingly [10].

\section{Patient Selection}

Patients were selected from the MATCH-study, an ongoing registration cohort including patients who undergo curative resection for primary CRC in 7 centres in the Rotterdam region, the Netherlands (Institutional Review Board number MEC-2007-
088). Patients with colon cancer included in the first 5 years of the MATCH-study (July 1, 2007-July 1, 2012) were selected for this study if they were willing to give written informed consent.

\section{Follow-Up}

The surveillance of patients after curative colon resection in the Netherlands is performed by gastrointestinal surgeons, alone or in collaboration with specialized NPCs. Patients treated with adjuvant chemotherapy are referred to the surgeon for follow-up as well. The surveillance program includes visits to the outpatient clinic every 3-6 months in the first 3 years and yearly visits up to 5 years after surgery. CEA blood levels are checked every 3 months in the first 3 years and every 6 months in years 4 and 5. Imaging of the liver (ultrasound or CT) is performed every 6 months in the first 2 years and on a yearly basis up to 5 years after surgery. A colonoscopy check is performed 1 year after surgery; thereafter the colonoscopy surveillance guidelines are followed (Table 1) [11, 12].

Follow-up visits were scored using patient charts and an electronic appointment history log. A follow-up visit consisted of an appointment with a surgeon or a trained NPC specialized in oncology. Additional, CEA testing with an abdominal ultrasound or abdominal CT scanning was performed.

Patients were divided into groups based on the number of visits in the first year using the national guideline for the follow-up of patients after curative surgery for CRC, in which 4 visits with a minimum of 2 visits in the first year is advised [13]. Patients with 3 or more visits in the first year were classified as "intensive" and patients with 2 or fewer visits were classified as "minimal." The involvement of an NPC in the follow-up was scored per centre (i.e., all patients from centres in which an NPC was present were categorized in the NPC group).

Information about whether disease recurrence was found in symptomatic or asymptomatic patients was registered. Recurrence was found either because of a regular follow-up visit or as a result of additional imaging in the context of complaints. Furthermore, the number of metastatic sites, the treatment modality, as well as the curative or palliative intent of the treatment plan was studied.

\section{Statistical Analysis}

Summary data for the study groups was presented as frequencies and percentages. Continuous variables were presented as mean values with SDs. The difference in distribution between
18

Dig Dis 2018;36:17-25

DOI: $10.1159 / 000478848$
Coebergh van den Braak/Lalmahomed/ Büttner/Hansen/Ijzermans 
groups was determined using a $\chi^{2}$-test. Differences in continuous variables were determined using a Student $t$ test. The univariable and multivariable survival analyses were performed using Cox regression models with centre included as strata to adjust for centre heterogeneity. Kaplan-Meier estimates were used to visualize overall effects. Disease-free survival (DFS) was defined as the time elapsed between the date of surgery and either the date of any recurrence of disease or the date of the last follow-up visit at which a patient was considered to have no recurrence. OS was defined as the time elapsed between the date of surgery and either the date of death or the date of the last check in the Municipal Personal Records Database at which a patient was censored. Survival after recurrence (SAR) was defined as the time elapsed between the date of recurrence of disease and either the date of death or the date of the last check in the Municipal Personal Records Database at which a patient was censored. All statistical analyses were performed using SPSS version 21 (IBM, Armonk, NY, USA). Significance was determined by a $p$ value $<0.05$.

\section{Results}

\section{Patient Characteristics}

A total of 870 patients were enrolled in the MATCH-study from July 1, 2007 to July 1, 2012. Of these patients, 71 patients died within the first year after surgery and 118 patients were lost to follow-up in the first year, resulting in a study sample size of $681 \mathrm{pa}-$ tients.

The baseline characteristics are summarized in Table 2. Most of the patients were in the "intensive" group $(79.9 \%)$. The mean age of the total population was 69.3 years. Just over half of the patients were male (54.0\%). Although the distribution of the ASA classification did not differ between the 2 follow-up groups, significantly more patients in the "intensive" group underwent a laparoscopic procedure compared to the "minimal" group (56.1 vs. $44.1 \%, p=0.01$, respectively). Radicality was similar in both groups (both $>99 \%$ ). Just over half of the patients had a left-sided tumour (53.3\%). Significant differences were seen in the distribution of both tumour stage and tumour grade between the 2 groups, with fewer stage III patients (19.5 vs. $29.2 \%$, $p=0.046)$ and more moderately differentiated tumours ( 77.6 vs. $67.4 \%, p=0.04$ ) in the "intensive" group. The majority of tumours showed no angioinvasive growth (91.5\%). The percentage of patients receiving adjuvant therapy was $24.0 \%$ for the whole group, and was significantly related to the then existing tumour stage (stage I $6.2 \%$, stage II $14.5 \%$ and stage III $70.3 \%, p=7 \times 10^{-49}$ ). Most patients had no recurrence of disease within the follow-up period (87.5\%).

Nonphysician Clinicians Add Value in CRC Follow-Up

\section{Nonphysician Clinicians}

An NPC was involved in the follow-up of 394 (57.9\%) patients after curative resection in 4 of the 7 participating centres during the full study period. Patients with a followup in an outpatient clinic with an NPC did not significantly differ from patients followed up in an outpatient clinic without an NPC for any of the basic characteristics except for ASA $(p=0.002)$ and tumour grade $(p=0.00002$; Table 3). Patients visiting an outpatient clinic working with an NPC were more often stratified to the "intensive" group compared to patients visiting an outpatient clinic without an NPC (84.3 vs. $73.9 \%, p=0.001)$.

\section{Survival}

In total, 85 (12.5\%) patients had recurrence of disease after curative surgery of the primary colon cancer. The median follow-up for DFS in the entire group was 34.3 months (interquartile range 20.1-45.8 months). Univariable cox regression analysis showed no significant difference in DFS between the "intensive" and "minimal" group (hazard ratio [HR] 1.50; 95\% CI 0.92-2.46, $p=0.1$; Fig. 1).

In total, 139 (20.4\%) patients died. The median followup for OS was 67.9 months (interquartile range 51.4-80.0 months). Univariable cox regression analysis, with centre included as strata to adjust for centre heterogeneity, showed a significant OS benefit for the "intensive" group compared to the "minimal" group (HR 1.87; 95\% CI $1.29-2.72, p=0.001$; Fig. 2 ), and showed a significant association between OS and age, ASA classification, the type of surgical procedure, side of the cancer, tumour stage and angioinvasive growth. In the conditional backward multivariable cox regression model including gender, ASA classification, surgical procedure, side of the cancer, tumour stage, tumour grade, angioinvasive growth and adjuvant therapy, and again including centre as strata to adjust for centre heterogeneity, led to a similar OS benefit for patients in the "intensive" group compared to the "minimal" group (HR 1.70; 95\% CI 1.11-2.60, $p=$ 0.01 ; Table 4).

For 74 of the 85 patients (87.1\%) with recurrence of disease, the reason for the disease recurrence (complaints or during a follow-up visit) could be determined. Significantly more patients in the "complaints" group had metastases at more than one anatomical site at the time of recurrence compared to the "follow-up" group (73.7 vs. $47.1 \%, p=0.04)$. The number of patients treated with curative intent did not significantly differ between the "follow-up" group and the "complaints" group (25.0 vs. $11.8 \%, p=0.2)$. However, patients in the "follow-up"

Dig Dis 2018;36:17-25

DOI: $10.1159 / 000478848$ 
Table 2. Baseline characteristics

\begin{tabular}{|c|c|c|c|c|}
\hline & $\begin{array}{l}\text { All } \\
(n=681)\end{array}$ & $\begin{array}{l}\text { Intensive } \\
(n=544)\end{array}$ & $\begin{array}{l}\text { Minimal } \\
(n=137)\end{array}$ & $p$ value \\
\hline Age, years, mean (SD) & $69.3(10.7)$ & $68.9(10.5)$ & $70.9(11.5)$ & 0.06 \\
\hline Gender, $n(\%)$ & & & & 0.6 \\
\hline Female & $313(46.0)$ & $253(46.5)$ & $60(43.8)$ & \\
\hline Male & $368(54.0)$ & $291(53.5)$ & $77(56.2)$ & \\
\hline $\mathrm{ASA}^{\mathrm{a}}, n(\%)$ & & & & 0.4 \\
\hline $\mathrm{I}$ & $94(17.2)$ & $70(16.0)$ & $24(21.8)$ & \\
\hline II & $376(68.7)$ & $307(70.3)$ & $69(62.7)$ & \\
\hline III & $75(13.7)$ & $58(13.3)$ & $17(15.5)$ & \\
\hline IV & $2(0.4)$ & $2(0.5)$ & $0(0)$ & \\
\hline Surgical procedure ${ }^{\mathrm{b}}, n(\%)$ & & & & 0.01 \\
\hline Open & $313(46.3)$ & $237(43.9)$ & $76(55.9)$ & \\
\hline Laparoscopic & $363(53.7)$ & $303(56.1)$ & $60(44.1)$ & \\
\hline Radicality ${ }^{\mathrm{c}}, n(\%)$ & & & & 0.8 \\
\hline Yes & $675(99.8)$ & $539(99.4)$ & $136(99.3)$ & \\
\hline No & $4(0.2)$ & $3(0.6)$ & $1(0.7)$ & \\
\hline Side of cancer, $n(\%)$ & & & & 0.7 \\
\hline Right & $318(46.7)$ & $252(46.3)$ & $66(48.2)$ & \\
\hline Left & $363(53.3)$ & $292(53.7)$ & $71(51.8)$ & \\
\hline TNM stage, $n(\%)$ & & & & 0.046 \\
\hline 1 & $196(28.8)$ & $161(29.6)$ & $35(25.5)$ & \\
\hline 2 & $339(49.8)$ & $277(50.9)$ & $62(45.3)$ & \\
\hline 3 & $146(21.4)$ & $106(19.5)$ & $40(29.2)$ & \\
\hline Tumour grade $^{\mathrm{d}}, n(\%)$ & & & & 0.04 \\
\hline Good & $84(12.6)$ & $63(11.8)$ & $21(15.9)$ & \\
\hline Moderate & $505(75.6)$ & $416(77.6)$ & $89(67.4)$ & \\
\hline Poor & $65(9.7)$ & $49(9.1)$ & $16(12.1)$ & \\
\hline Other & $14(2.1)$ & $8(1.5)$ & $6(4.5)$ & \\
\hline Angioinvasive growth, $n(\%)$ & & & & 0.6 \\
\hline No & $623(91.5)$ & $499(91.7)$ & $124(90.5)$ & \\
\hline Yes & $58(8.5)$ & $45(8.3)$ & $13(9.5)$ & \\
\hline Adjuvant therapy ${ }^{\mathrm{e}}, n(\%)$ & & & & 0.04 \\
\hline No & $516(76.0)$ & $421(77.7)$ & $95(69.3)$ & \\
\hline Yes & $163(24.0)$ & $121(22.3)$ & $42(30.7)$ & \\
\hline Recurrence, $n(\%)$ & & & & 0.2 \\
\hline No & $596(87.5)$ & $481(88.4)$ & $115(83.9)$ & \\
\hline Yes & $85(12.5)$ & $63(11.6)$ & $22(16.1)$ & \\
\hline
\end{tabular}

group did have a significantly better SAR compared to patients in the "complaints" group (HR 3.60; 95\% CI 1.92-6.74, $p=0.00006$; Fig. 3).

\section{Discussion and Conclusions}

The frequency of follow-up of patients after curative surgery of CRC and the tests to be used during follow-up still is a topic of debate. Current literature suggests an intensive follow-up with CEA measurements and/or CT imaging. Due to the labour intensity of the follow-up, NPCs are increasingly employed for the follow-up of these patients. However, no literature has been published assessing the added value of an NPC in the follow-up.

In our cohort, significantly more patients seen in an outpatient clinic with an NPC were categorized in the "intensive" group compared to patients followed up in an outpatient clinic without an NPC (84.3 vs. $73.9 \%, p=$ 0.001). Patients in the "intensive" group had a similar DFS but a significantly better OS compared to patients in the "minimal" group, even when adjusting for other prog- 
Table 3. Characteristics for NPC present or not present

\begin{tabular}{|c|c|c|c|}
\hline & $\begin{array}{l}\text { NPC not } \\
\text { present } \\
(n=287)\end{array}$ & $\begin{array}{l}\text { NPC } \\
\text { present } \\
(n=394)\end{array}$ & $p$ value \\
\hline Age, years, mean (SD) & $69.1(10.7)$ & $69.4(10.8)$ & 0.9 \\
\hline Gender, $n(\%)$ & & & 0.5 \\
\hline Female & $136(47.4)$ & $177(44.9)$ & \\
\hline Male & $151(52.6)$ & $217(55.1)$ & \\
\hline $\mathrm{ASA}^{\mathrm{a}}, n(\%)$ & & & 0.002 \\
\hline I & $61(23.5)$ & $33(11.5)$ & \\
\hline II & $160(61.5)$ & $216(75.3)$ & \\
\hline III & $38(14.6)$ & $37(12.9)$ & \\
\hline IV & $1(0.4)$ & $1(0.3)$ & \\
\hline Surgical procedure ${ }^{\mathrm{b}}, n(\%)$ & & & 0.4 \\
\hline Open & $126(44.4)$ & $187(47.7)$ & \\
\hline Laparoscopic & $158(55.6)$ & $205(52.3)$ & \\
\hline Radicality $^{\mathrm{c}}, n(\%)$ & & & 0.09 \\
\hline Yes & $0(0)$ & $4(1.0)$ & \\
\hline No & $287(100)$ & $388(99.0)$ & \\
\hline Side of cancer, $n(\%)$ & & & 0.09 \\
\hline Right & $123(42.9)$ & $195(49.5)$ & \\
\hline Left & $164(57.1)$ & $199(50.5)$ & \\
\hline TNM stage, $n(\%)$ & & & 0.6 \\
\hline 1 & $80(27.9)$ & $116(29.4)$ & \\
\hline 2 & 149 (51.9) & $190(48.2)$ & \\
\hline 3 & $58(20.2)$ & $88(22.3)$ & \\
\hline Tumour grade ${ }^{\mathrm{d}}, n(\%)$ & & & 0.00002 \\
\hline Good & $48(17.0)$ & $36(9.3)$ & \\
\hline Moderate & $186(66.0)$ & $319(82.6)$ & \\
\hline Poor & $39(13.8)$ & $26(6.7)$ & \\
\hline Other & $9(3.2)$ & $5(1.3)$ & \\
\hline $\begin{array}{l}\text { Angioinvasive growth, } \\
n(\%)\end{array}$ & & & 0.7 \\
\hline No & $261(90.9)$ & $362(91.9)$ & \\
\hline Yes & $26(9.1)$ & $32(8.1)$ & \\
\hline Adjuvant therapy ${ }^{\mathrm{e}}, n(\%)$ & & & 0.6 \\
\hline No & $221(77.0)$ & $295(75.3)$ & \\
\hline Yes & $66(23.0)$ & $97(24.7)$ & \\
\hline Recurrence, $n(\%)$ & & & 0.1 \\
\hline No & $245(85.4)$ & $351(89.1)$ & \\
\hline Yes & $42(14.6)$ & 43 (10.9) & \\
\hline
\end{tabular}

nostic factors (HR 1.71; 95\% CI 1.12-2.62). Finally, patients in the "follow-up" group less often had metastases at more than one anatomical site at the time of recurrence ( 47.1 vs. $73.7 \%, p=0.04$ ), and a better SAR compared to the "complaints" group (HR 3.60; 95\% CI 1.92-6.74).

Our study supports the added value of NPCs in the follow-up of patients after curative surgery for CRC to improve adherence to the surveillance program. Nonetheless, its effect on DFS and OS remains to be determined.

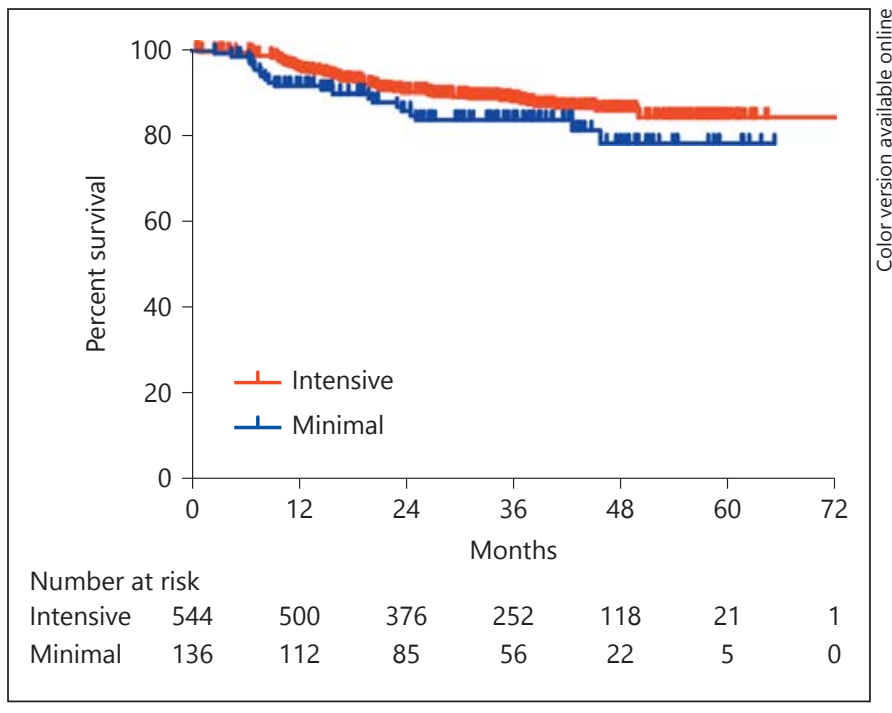

Fig. 1. Kaplan-Meier curve of the disease-free survival for the "intensive" and "minimal" group.

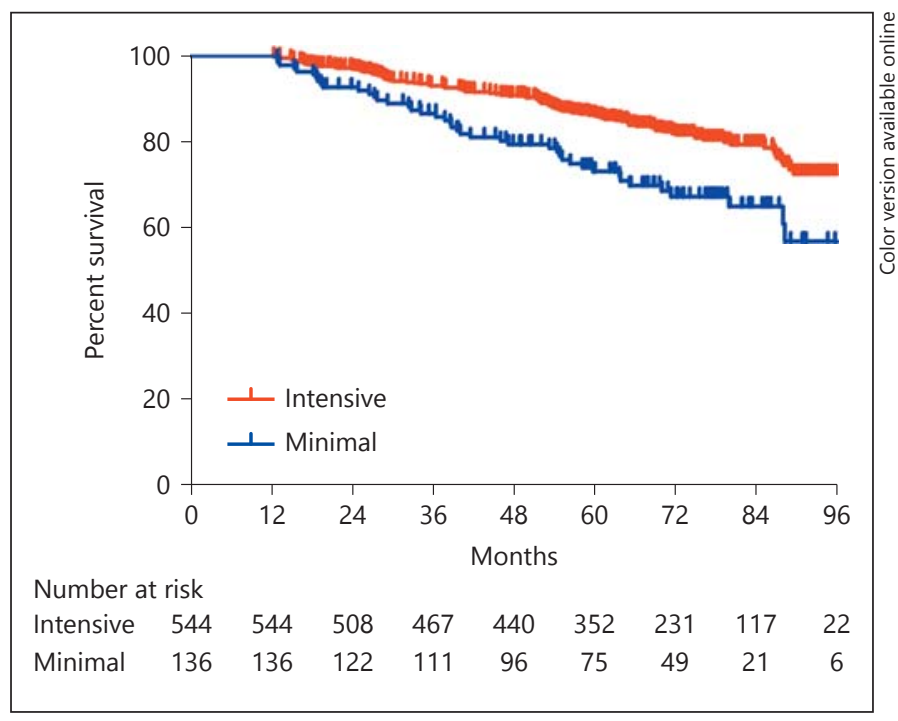

Fig. 2. Kaplan-Meier curve of the overall survival for the "intensive" and "minimal" group.

This study has several limitations. First, no detailed information on the actual attendance of the NPCs during the follow-up visits was available. Although all follow-up visits were documented in the patient charts, these may have been conducted by either the NPC or the treating specialist. Yet, the follow-up visits alone probably do not accurately reflect the role of the NPC. In practice, they also make sure that a patient seen by a specialist receives an invitation for the next visit, including the appropriate 
Fig. 3. Kaplan-Meier curve of the survival after recurrence for the "complaints" and "follow-up" group.

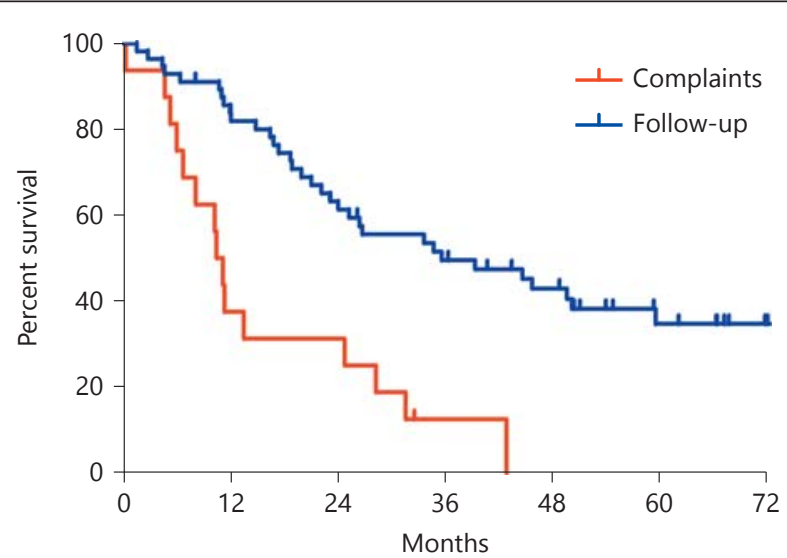

Number at risk

Follow-up $\begin{array}{lllllll}54 & 43 & 31 & 24 & 19 & 10 & 2\end{array}$

Table 4. Univariable and multivariable cox regression model for OS with centre included as strata to adjust for centre heterogeneity

\begin{tabular}{|c|c|c|c|c|}
\hline & Univariable & $p$ value & Multivariable & $p$ value \\
\hline \multicolumn{5}{|l|}{ Follow-up group } \\
\hline Intensive & 1 & & 1 & \\
\hline Minimal & $1.87(1.29-2.72)$ & 0.001 & $1.70(1.11-2.60)$ & 0.01 \\
\hline Age, years & $1.08(1.06-1.10)$ & $8 \times 10^{-15}$ & $1.08(1.06-1.11)$ & $7 \times 10^{-10}$ \\
\hline \multicolumn{5}{|l|}{ Gender, $n(\%)$} \\
\hline Female & 1 & & & \\
\hline Male & $1.16(0.83-1.63)$ & 0.4 & & \\
\hline \multicolumn{5}{|l|}{$\mathrm{ASA}^{\mathrm{a}}, n(\%)$} \\
\hline $\mathrm{I}$ & 1 & 0.0000006 & 1 & 0.008 \\
\hline II & $2.71(1.35-5.44)$ & & $1.38(0.65-2.91)$ & \\
\hline III & $6.70(3.16-14.18)$ & & $2.44(1.08-5.52)$ & \\
\hline IV & $13.76(1.59-118.93)$ & & $14.02(1.46-134.98)$ & \\
\hline \multicolumn{5}{|c|}{ Surgical procedure ${ }^{\mathrm{b}}, n(\%)$} \\
\hline Open & 1 & & & \\
\hline Laparoscopic & $0.65(0.46-0.93)$ & 0.02 & & \\
\hline \multicolumn{5}{|l|}{ Radicality ${ }^{\mathrm{c}}, n(\%)$} \\
\hline Yes & 1 & & & \\
\hline No & $20.59(0.0004-1.1 \times 10 \mathrm{E} 6)$ & 0.6 & & \\
\hline \multicolumn{5}{|c|}{ Side of cancer, $n(\%)$} \\
\hline Right & 1 & & & \\
\hline Left & $0.69(0.49-0.96)$ & 0.03 & & \\
\hline \multicolumn{5}{|l|}{ TNM stage, $n(\%)$} \\
\hline 1 & 1 & 0.005 & 1 & 0.0003 \\
\hline 2 & $1.32(0.86-2.03)$ & & $1.45(0.89-2.36)$ & \\
\hline 3 & $2.11(1.32-3.37)$ & & $3.26(1.80-5.89)$ & \\
\hline \multicolumn{5}{|c|}{ Tumour grade ${ }^{\mathrm{d}}, n(\%)$} \\
\hline Good & 1 & 0.2 & & \\
\hline Moderate & $0.91(0.51-1.63)$ & & & \\
\hline Poor & $1.49(0.74-2.98)$ & & & \\
\hline Other & $1.42(0.46-4.36)$ & & & \\
\hline \multicolumn{5}{|c|}{ Angioinvasive growth, $n(\%)$} \\
\hline No & 1 & & 1 & \\
\hline Yes & $2.09(1.30-3.35)$ & 0.002 & $1.98(1.15-3.39)$ & 0.01 \\
\hline \multicolumn{5}{|c|}{ Adjuvant therapy ${ }^{\mathrm{e}}, n(\%)$} \\
\hline No & 1 & & & \\
\hline Yes & $0.69(0.45-1.06)$ & 0.09 & & \\
\hline
\end{tabular}

${ }^{\mathrm{a}} n=134 ;{ }^{\mathrm{b}} n=5 ;{ }^{\mathrm{c}} n=2 ;{ }^{\mathrm{d}} n=13 ;{ }^{\mathrm{e}} n=2$. 
tests. Therefore, we chose to include the presence of an NPC in a participating centre in our analyses. If deemed useful, the effect of the "behind-the-scenes" actions should be assessed prospectively, since they cannot be reliably recalled from the patient charts. Second, the first year after resection was used to divide the patients into the 2 groups. Therefore, all patients who died within the first year were excluded and thus no conclusions can be drawn for that selected group of patients. Patients who died in the first year had a higher mean age, a higher ASA III score, more open surgery, less often adjuvant chemotherapy and more frequent disease recurrence compared to the included patients (data not shown). However, given the short time period after resection in which these events occurred, the impact of the follow-up is likely to be minimal. Third, the study cohort was derived from an observational study cohort. Therefore, patients at risk for recurrence may be intentionally followed more intensively than patients at low risk for recurrence, that is, bias by indication. However, the groups did not significantly differ in terms of age, gender, location of the tumour, TNM stage or total recurrences. Last, the differences in frequency of the follow-up visits might have resulted in lead time bias. However, the average difference in the interval between visits is small (3 months), and lead time bias would have overestimated the DFS in the "minimal" group, which would not explain the differences found in OS. Furthermore, lead time bias may also account for part of the observed survival difference between the "follow-up" and the "complaints" group. However, the difference in median survival time (35.5 vs. 10.4 months) is not likely to be explained by a potential lead time bias with an average interval between visits of 3 months. Furthermore, similar survival estimates are observed when stratifying patients to "one metastatic site" and "more than one metastatic site" (data not shown), suggesting that the observed effect may largely be explained by the disease burden at the time of recurrence.

NPCs are progressively involved in medical work [14]. They are appointed for both in hospital as well as outpatient clinic tasks, ranging from invasive diagnostic procedures to consultation. For instance, literature has reported that invasive procedures like the placement of intracranial pressure monitors, ultrasound-assisted percutaneous liver biopsy and dermatologic surgical procedures are both feasible and safe in the hands of trained NPCs [15-17]. In the field of CRC, training programs for flexible sigmoidoscopy in the context of screening have been set up and are currently being evaluated [18-20]. Interestingly, a survey conducted in 2000 among 1,900 health care professionals showed that more than half of

Nonphysician Clinicians Add Value in CRC Follow-Up the physicians involved in CRC were reluctant to let NPCs perform these procedures [21]. Therefore, future efforts should not only be focused on training programs for the NPCs but also on understanding and resolving the reasons for these reluctances. Although experience in the clinical practice suggests the existence of an added value of involving NPCs in the follow-up after curative colorectal surgery, literature supporting these suggestions is scarce. In the field of cardiology, Albert et al. [22] published an article describing an increased adherence to the guideline-recommended heart failure therapies when employing NPCs. This study supports the concept that NPCs may have an important role in the follow-up of patients with CRC as adherence is improved and the surgeon's workload is reduced.

Although follow-up after curative surgery for CRC is considered effective, both the tests used during follow-up and the frequency of the follow-up are still under debate. Meta-analyses have shown that CEA measurement and CT imaging are the only diagnostic tests with significant potential to detect curatively treatable metastatic recurrence in patients with CRC $[7,8]$. However, although the sensitivity of ultrasound is inferior to the sensitivity of CT imaging at a patient level (73 vs. $84 \%$ ), ultrasound in clinical practice is used as an alternative for CT imaging for the detection of liver metastasis $[4,23]$. The recently published FACS trial, a prospective randomized controlled trial compared no follow-up with 3 intervention arms with CEA follow-up, CT follow-up, or CEA + CT follow-up. They showed that significantly more patients with recurrence of disease in all 3 intervention arms were treated with curative intent, although the number of recurrences and the time of detection of the recurrence did not significantly differ from the control group. However, no survival benefit was found for any of the intervention arms compared to the control group [3]. Following these findings, only follow-up visits during which any combination of CEA testing, an abdominal ultrasound and an abdominal CT-scan were performed, were taken into account in our study. Follow-up visits with an abdominal ultrasound were also included in our study, since it is generally accepted as imaging modality to assess the presence of colorectal liver metastases. Patients in the "intensive" group had a similar DFS but a significantly better OS compared to patients in the "minimal" group even when adjusting for other prognostic factors (HR 1.71; 95\% CI 1.12-2.62). Interestingly, significantly more patients in the "complaints" group had metastases at more than one anatomical site at the time of recurrence compared to the "follow-up" group (47.1 vs. $73.7 \%, p=$ $0.04)$. Patients in the "follow-up" group were not signifi-

Dig Dis 2018;36:17-25

DOI: $10.1159 / 000478848$ 
cantly more often treated with curative intent compared to the patients in the "complaints" group. However, patients in the "follow-up" group had a significantly better SAR compared to the patients in the "complaints" group (HR 3.60; 95\% CI 1.92-6.74), which may be explained by the difference in disease burden at the time of recurrence.

In conclusion, our data indicates that hospitals having an NPC have a better adherence to the surveillance program for patients resected for CRC. Overall, patients in surveillance programs do have less multifocal disease recurrence and a better 3-year SAR, which substantiates the beneficial effect of this approach.

\section{Acknowledgement}

The authors thank de MATCH study group consisting of: Peter-Paul L.O. Coene, MD, PhD, Department of Surgery, Maasstad Hospital, Rotterdam, The Netherlands; Jan Willem T. Dekker, MD, PhD, Department of Surgery, Reinier de Graaf Hospital, Delft, The Netherlands; David Zimmerman, MD, PhD, ElisabethTweesteden Hospital, Tilburg, The Netherlands; Geert W.M. Tetteroo, MD, PhD, Department of Surgery, IJsselland Hospital, Capelle a/d IJssel, The Netherlands; Wouter J. Vles, MD, PhD, Department of Surgery, Ikazia Hospital, Rotterdam, The Netherlands; and Wietske W. Vrijland, MD, Department of Surgery, Sint Franciscus Hospital, Rotterdam, The Netherlands.

The authors thank the NPCs Lidy van Driel and Trudy Rapmund (Department of Surgery, Maasstad Hospital, Rotterdam, The Netherlands), Sandra de Bruijn and Erlinde de Graaf (Depart- ment of Surgery, Reinier de GraafHospital, Delft, The Netherlands), Walther Jansen and Monique Borremans (Department of Surgery, Elisabeth-Tweesteden Hospital, Tilburg, The Netherlands), Jaco Zwijnenburg and Meanne Voogt (Department of Surgery, IJsselland Hospital, Capelle a/d IJssel, The Netherlands), Esther Schmidt and Hanneke Kreiter (Department of Surgery, Ikazia Hospital, Rotterdam, The Netherlands), and Annemarie van der Spek (Department of Surgery, Sint Franciscus Hospital, Rotterdam, The Netherlands) for the inclusion of patients in the MATCH study.

\section{Ethical Statement}

All subjects gave informed consent and the study protocol was approved by the Medical Ethical Committee of the Erasmus MC (MEC-2007-088).

\section{Disclosure Statement}

The authors declare no conflicts of interest.

\section{Author Contribution}

R.R.J.C.B., Z.S.L., and S.B. have acquired, analysed and interpreted the data, drafted the article and approved the final version; B.E.H. has analysed the data, critically revised the article and approved of the final version; J.N.M.I. has designed the study, critically revised the article and approved of the final version.

\section{References}

1 UK Cancer Research: Cancer StatisticsColorectal Cancer, 2013.

-2 Elferink MA, de Jong KP, Klaase JM, Siemerink EJ, de Wilt JH: Metachronous metastases from colorectal cancer: a population-based study in north-east netherlands. Int J Colorectal Dis 2015;30:205-212.

- 3 Primrose JN, Perera R, Gray A, Rose P, Fuller A, Corkhill A, George S, Mant D; FACS Trial Investigators: Effect of 3 to 5 years of scheduled CEA and CT follow-up to detect recurrence of colorectal cancer: the FACS randomized clinical trial. JAMA 2014;311: 263-270.

4 Kievit J: Follow-up of patients with colorectal cancer: numbers needed to test and treat. Eur J Cancer 2002;38:986-999.

$\checkmark 5$ de Haas RJ, Wicherts DA, Flores E, Azoulay $\mathrm{D}$, Castaing D, Adam R: R1 resection by necessity for colorectal liver metastases: is it still a contraindication to surgery? Ann Surg 2008; 248:626-637.

6 Kanas GP, Taylor A, Primrose JN, Langeberg WJ, Kelsh MA, Mowat FS, Alexander DD, Choti MA, Poston G: Survival after liver re- section in metastatic colorectal cancer: review and meta-analysis of prognostic factors. Clin Epidemiol 2012;4:283-301.

7 Renehan AG, Egger M, Saunders MP, O'Dwyer ST: Impact on survival of intensive follow up after curative resection for colorectal cancer: systematic review and meta-analysis of randomised trials. BMJ 2002; 324:813.

8 Jeffery M, Hickey BE, Hider PN: Follow-up strategies for patients treated for non-metastatic colorectal cancer. Cochrane Database Syst Rev 2007;1:CD002200.

-9 Pita-Fernandez S, Alhayek-Ai M, GonzalezMartin C, Lopez-Calvino B, Seoane-Pillado T, Pertega-Diaz S: Intensive followup strategies improve outcomes in nonmetastatic colorectal cancer patients after curative surgery: a systematic review and meta-analysis. Ann Oncol 2015;26:644656.

10 von Elm E, Altman DG, Egger M, Pocock SJ, Gotzsche PC, Vandenbroucke JP; STROBE Initiative: The strengthening the reporting of observational studies in epidemiology
(STROBE) statement: guidelines for reporting observational studies. Lancet 2007;370: 1453-1457.

11 Tumors: Oncoline, Netherlands Comprehensive Cancer Organisation, 2017.

12 Gastroenterology: Dutch Guideline for Coloscopy Surveillance, Dutch Society of Gastroenterology, 2017.

13 Oncoline, 2014

14 Hing E, Uddin S: Physician Assistant and Advance Practice Nurse Care in Hospital Outpatient Departments: United States, 2008-2009. NCHS Data Brief, 2011, pp 1-8.

15 Young PJ, Bowling WM: Midlevel practitioners can safely place intracranial pressure monitors. J Trauma Acute Care Surg 2012;73: 431-434.

16 Gunneson TJ, Menon KV, Wiesner RH, Daniels JA, Hay JE, Charlton MR, Brandhagen DJ, Rosen CB, Porayko MK: Ultrasound-assisted percutaneous liver biopsy performed by a physician assistant. Am J Gastroenterol 2002;97:14721475. 
17 Tierney EP, Hanke CW, Kimball AB: Practice models and roles of physician extenders in dermatologic surgery. Dermatol Surg 2011; 37:677-683.

-18 Redwood D, Joseph DA, Christensen C, Provost E, Peterson VL, Espey D, Sacco F: Development of a flexible sigmoidoscopy training program for rural nurse practitioners and physician assistants to increase colorectal cancer screening among alaska native people. J Health Care Poor Underserved 2009;20:1041-1048.

19 Sansbury LB, Klabunde CN, Mysliwiec P, Brown ML: Physicians' use of nonphysician healthcare providers for colorectal cancer screening. Am J Prev Med 2003;25:179-186.
20 Horton K, Reffel A, Rosen K, Farraye FA: Training of nurse practitioners and physician assistants to perform screening flexible sigmoidoscopy. J Am Acad Nurse Pract 2001;13: 455-459.

21 Shaheen NJ, Crosby MA, O’Malley MS, Murray SC, Sandler RS, Galanko JA, Ransohoff DF, Klenzak JS: The practices and attitudes of primary care nurse practitioners and physician assistants with respect to colorectal cancer screening. Am J Gastroenterol 2000;95: 3259-3265.
22 Albert NM, Fonarow GC, Yancy CW, Curtis AB, Stough WG, Gheorghiade M, Heywood JT, McBride M, Mehra MR, O'Connor CM, Reynolds D, Walsh MN: Outpatient cardiology practices with advanced practice nurses and physician assistants provide similar delivery of recommended therapies (findings from improve HF). Am J Cardiol 2010;105:17731779.

23 Niekel MC, Bipat S, Stoker J: Diagnostic imaging of colorectal liver metastases with CT, MR imaging, FDG PET, and/or FDG PET/ CT: a meta-analysis of prospective studies including patients who have not previously undergone treatment. Radiology 2010;257:674684 . 